Silbers durch Bestimmung des Metallgehaits der kolloiden Lösung ermittelt.

Man vermischte je 5 Teile des Carragheenschleims mit je 2,5 Teilen Silbernitratlösung verschiedener Normalität und ließ die Flüssigkeiten je 3 Stunden lang unter gelegentlichem Umschütteln auf dem Wasserbade stehen. Man gewann übereinstimmend braune Systeme, die nun sogleich in gewässerte Pergamentschläuche übergefüllt und so lange gegen täglich dreimal erneuertes Wasser dialysiert wurden, bis im Aubenwasser eine Reaktion auf Silber- und Nitration nicht mehr festzustellen war. Alle Präparate überstanden die Dialyse gut und konnten daher aus den Schläuchen quantitativ zurückgewonnen werden.

Zur Bestimmung des Silbergehalts der kolIoiden Lösungen bedienten wir uns diesmal eines maBanalytischen Verfahrens, nachdem durch Vorversuche nachgewiesen worden war, daf Carragheenschleim sich durch wiederholtes Eindampfen mit konzentrierter Salpetersäure so weit zerstören läßt, daß ein Einflub auf die Volhard'sche Methode nicht nachweisbar ist. So wurden gemessene Mengen der dialysierten Flüssigkeiten im Porzellanschälchen mit konzentrierter Salpetersäure so oft eingedampft, bis auf Zusatz von etwas Wasser eine klare farblose Lösung entstand, und die schlieflich resultierende Flüssigkeit in bekannter Weise nach J. Volhard titriert.

Selbstverständlich sind wir uns darüber klar, daB die in Tab. II zusammengestellten und durch

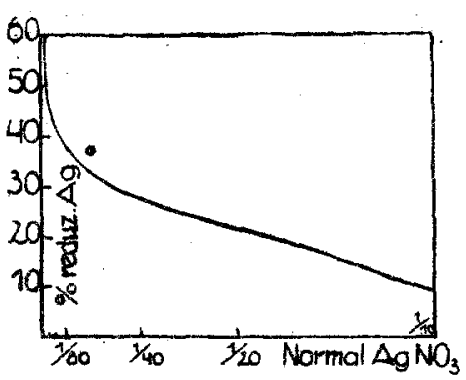

Fig. 1

Fig. 1 veranschaulichten Ergebnisse solcher Versuche im Hinblick auf das Verhalten von Carragheenschleim während der Dialyse niemals absolute Werte zu liefern imstande sind. $\mathrm{Zu}$ Vergleichszwecken aber sind sie brauchbar, und sie ergeben, wie auf Grund der präparativen Befunde zu erwarten stand, dab die Menge des durch die Schleimsubstanzen in der Wärme reduzierten Silbers mit sinkender Silberionenkonzentration der Ausgangslösungen zunimmt.

Tabelle II.

Normalität

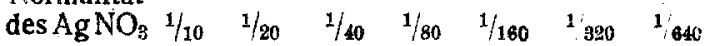

Proz. redu-

ziertes Ag $8,98 \quad 21,49 \quad 27,52 \quad 37,11 \quad 37,72 \quad 43,39 \quad 58,54$ ;

Da die Schutzwirkung des Carragheenschleims sich als durchaus zufriedenstellend erwiesen hat, gedenken wir noch weiter Versuche mit diesem Extrakte durchzuführen.

\title{
Die Elektroadsorption als rein chemische Erscheinung.
}

Von J. M. Kolt th off (Utrecht). (Eingegangen am 9. Oktober 1921.)

1. Nach dem bekannten Satze von Gibbs ist die Konzentration eines gelösten Stoffes an der Oberfläche kleiner als in der Flüssigkeit, wenn bei zunehmender Konzentration die Oberflächenspannung sich erhöht; wenn dagegen umgekehrt die Oberflächenspannung des Lösungsmittels durch den gelösten Stoff vermindert wird, häuft der letztere sich an der Oberflăchè an. Wenn man die Konzentrationsänderung an der Oberfläche mit Adsorption gleichstellt, dann ist diese positiv, wenn der gelöste Stoff die Oberflächenspannung erniedrigt und negativ im umgekehrten Falle. Die Aenderung in der Oberflächenspannung $1\left(\gamma-\gamma^{\prime}\right)$ wird beherrscht durch die Gleichung

$$
\left(\gamma-\gamma^{\prime}\right)=\alpha c^{1 / \mathrm{n}}
$$

(c ist die Konzentration, $\alpha$ und $1 / \mathrm{n}$ sind Konstanten).

In Verbindung mit der Definition der Adsorption ist es kein Wunder, dab die Adsorption von kapillaraktiven Stoffen durch eine analoge Gleichung beherrscht wird:

$$
\mathrm{x} / \mathrm{m}=\alpha \mathrm{c}^{1 / \mathrm{m}}
$$

$(\mathrm{x} / \mathrm{m}$ ist die Menge des adsorbierten Stoffes pro g Adsorbens).

Während wir uns also bei der Adsorption von kapillaraktiven Stoffen mit der physikalischen Erklärung einverstanden erklären können, geht das schwieriger bei der Adsorption von kapillarinaktiven Stoffen, wie vielen Elektrolyten. 
Freundlich') drückt sich hierüber wie folgt aus: "Die Angaben über die Adsorption von Salzen sind daher noch recht widerspruchsvoll und schwer zu deuten."

Merkwürdig beim Verhalten von Elektrolyten ist auberdem die Tatsache, dab ein so großer Unterschied zwischen der Adsorption von Kationen und Anionen besteht. So wird von einem Farbstoffsalz durch Kohle allein das Farbstoffion adsorbiert, das andere Kation oder Anion nicht. Von Alkaloidsalzen wird nur das Alkaloidion adsorbiert, usw. Da die Flüssigkeit elektrisch neutral bleiben muk, mub die Kohle also bei ihrer adsorbierenden Wirkung eine aquivalente Menge eines lons mit demselben Ladungszeichen als das adsorbierte in Lösung senden. Das adsorbierte Ion verdrängt also bei seiner Adsorption ein anderes lon von der Oberfläche der Kohle; man nennt eine derartige Adsorption eine verdrängende oder Ionen- oder Elektroadsorption.

Diese verdrängende Adsorption kann man nun als einen rein chemischen Vorgang ansehen; es ist unmöglich, die Tatsachen mit Hilfe der Oberflächenspannung physikalisch zu erklären. Diese verdrängende Adsorption muB wohl der Anwesenheit von Elektrolyten auf dern Adsorbens zugeschrieben werden. Bei der selektiven lonenadsorption wird ein anderes Ion in die Lösung gesandt. Bei Kohle kann das natürlich kein Kohlenstoffion sein. In Uebereinstimmung mit dem chemischen Vorgang steht die Tatsache, daB Kohle um so weniger von Elektrolyten "adsorbiert", je reiner sie ist. Zwar kann man nicht ungekehrt sagen, da Kohle um so besser adsorbiert, je unreiner sie ist, denn der Aschengehalt der Kohle spielt, wie aus der Untersuchung von $\mathrm{GlaB}$ n e r und $S$ uid a ${ }^{2}$ ) hervorgeht, bei der Adsorption nur eine geringe Rolle. Diese beiden Verfasser schreiben das Adsorptionsvermögen der Kohle hauptsächlich bestimmten Stickstoffverbindungen zu, die Derivate von Zyan (Melanide, Melan u. a.) sind. Sie weisen auch bereits darauf hin, daB gerade die Verunreinigungen der Kohle die Adsorption verursachen und daB man letztere nicht physikalisch erklären kann.

In Uebereinstimmung mit der chemischen Auffassung der Adsorption steht ferner, daB Kieselsäure nur basische Farbstoffe fixiert, Aluminiumoxydhydrat und Eisenoxydhydrat nur

1) H. F r e u d l i c h, Kapllarchemie (Leipzig 1909), 165.

2) Glabner u. Suida, Liebig's Ann. d. Chem. 357, 95 (1908). saure Parbstoffe. Auch hier ist die Adsorption wahrscheinlicb nur verdrängender Art. Wenn man z. B. Eisenoxyd aus Eisennitrat durch Glühen darstellt, dann wirkt es adsorbierend, so lange noch Nitrat daran gebunden ist. Die Adsorption eines Anions $A^{\prime}$ kann man dann wie folgt ausdriicken:

$$
\mathrm{Fe}(\mathrm{OH})_{2} \mathrm{NO}_{3}+\mathrm{A}^{*} \leftrightarrows \mathrm{Fe}_{2}(\mathrm{OH})_{2} \mathrm{~A}+\mathrm{NO}_{3}^{\prime}
$$

Glüht man das Präparat so lange, bis alles Nitrat verschwunden ist, dann wirkt es nicht mehr adsorbierend. Man muB also annehmen, dab das "adsorbierte" Ion chemisch durch die Verunreinigungen des Adsorbens festgelegt wird. Bei früheren Untersuchungen hatte Freund. Iich bereits gefunden, daß Farbstoffe an der Oberfläche der Kohle als unlösliche Lacke festgelegt werden, so dab sie mit verschiedenen Ausschüttelungsflüssigkeiten nicht mehr zu entziehen sind. Ferner ist es auffallig, dab beim Sättigungszustande des Adsorbens häufig aquivalente Mengen von Kationen bzw. Anionen adsorbiert sind.

Sodann läßt sich physikalisch nicht erklären, dab ein kapillaraktiver Stoff ohne Einflub auf die Adsorption eines Elektrolyten ist. Auch andere Tatsachen weisen noch darauf hin, dab an der Oberfläche des Adsorbens eine chemische Reaktion stattindet, wobei gerade die Verunreinigungen des Adsorbens das "adsorbierte* Ion fixieren.

2. Die Beziehung zwischen der Menge des adsorbierten Stoffes und dessen Endkonzentration in der Flüssigkeit wird auch bei der Elektroadsorption durch die Adsorptionsisotherme

$$
\frac{x}{m}=\alpha c^{1 / n}
$$

beherrscht. Wenn wir nun die "Adsorption" rein chemisch erkJären wollen, müssen wir die Adsorptionsisotherme auch mit Hilfe der Stöchiometrie ableiten können. Es muB dann vorausgeschickt werden, daB $\frac{x}{m}$, d. i. die Menge Stoff in Millimolen, die von einem Gramm Adsorbens an der Oberfläche festgelegt wird, natürlich auch gleich ist der Menge des verdrängten lons, das in die Flüssigkeit gesandt wird.

Um nun die Adsorptionsisotherme chemiscb abzuleiten, wollen wir zuerst vom einfachen Falle ausgehen, da wir ein schwerlösliches Salz AB mit einer Lösung schüttein, die Clonen enthält, wobei die schwerlösliche Verbindung $A C$ gebildet werden kann. Nehmen wir z. B. an, dab Silberbromid mit einer Lösung 
des einen oder anderen Rhodanides geschüttelt wird. Die eintretende Reaktion können wir durch die folgende Gleichung ausdrücken:

$$
\mathrm{AgBr}+\mathrm{CNS}^{\prime} \leftrightarrows \mathrm{AgCNS}+\mathrm{Br}^{\prime} \text {. }
$$

Wenn $I_{A g B r}$ das Ionenprodukt von Sillberbromid bedeutet, und $I_{A g} C N$ das von Silberrhodanid, dann ist

$$
[\mathrm{Ag}]=\frac{l_{\mathrm{Ag} B r}}{\left[\mathrm{Br}^{\prime}\right]}=\frac{I_{\mathrm{Ag} \mathrm{CNS}}}{[\mathrm{CNS}]}
$$

Hieraus läBt sich direkt ableiten, daB

$$
\left[\mathrm{Br}^{\prime}\right]=\frac{I_{\text {AgBr}}}{I_{\text {AgCN }}} \cdot\left[\mathrm{CNS}^{\prime}\right]=\mathrm{K}\left[\mathrm{CNS}^{\prime}\right] .
$$

Nun ist $\left[\mathrm{Br}^{\circ}\right]$ gleich der Menge Bromionen, die in die Lösung gesandt werden, also auch gleich der Menge CNS', die festgelegt werden, oder in unserem Falle $\frac{x}{m}{ }^{3}$ ), während [CNS gleich der Endkonzentration c des festgelegten lons ist. Obengenannte Gleichung (1) können wir also auch in folgender Form schreiben:

$$
\frac{\mathrm{x}}{\mathrm{m}}=\mathrm{Kc} \text {. }
$$

Wenn wir nun an Stelle von $\mathrm{AgBr}$ einmal $\mathrm{Ag}_{2} \mathrm{~S} \mathrm{O}_{4}$ nehmen und dieses mit einer Chloridlösung schütteln, dann wird die eintretende Reaktion durch die folgende Gleichung ausgedrụ̈ckt:

$$
\mathrm{Ag}_{2} \mathrm{SO}_{4}+2 \mathrm{Cl}^{\prime} \leftrightarrows 2 \mathrm{AgCl}+\mathrm{SO}_{4}^{\prime \prime} \text {. }
$$

Wir können dann ableiten, daß

$$
\left[\mathrm{SO}_{4}{ }^{\prime \prime}\right]=\frac{I_{\mathrm{Ag}_{9} \mathrm{SO}_{4}}}{I_{\mathrm{Ag}_{\mathrm{g}} \mathrm{Cl}}} \cdot\left[\mathrm{Cl}^{\prime}\right]^{2}=\mathrm{K}\left[\mathrm{Cl}^{\prime}\right]^{2}
$$

$$
\text { oder } \quad \frac{\mathrm{x}}{\mathrm{m}}=\mathrm{Kc} \mathrm{c}^{2} \text {. }
$$

Wenn wir nun den allgemeinen Fall nehmen, daB der schwerlösliche Stoff $A_{x} B_{y}$ mit einer Lösung geschüttelt wird, die $C^{\prime}$ enthält, wobei die schwerlossliche Verbindung $A_{p} C_{z}$ gebildet wird, dann können wir die Reaktion auf folgende Weise ausdrücken:

$$
\text { a } A_{x} B_{y}+b C^{\prime} \stackrel{\leftarrow}{\longleftrightarrow} A_{p} C_{z}+y^{\prime}
$$

Dann ist

$$
\left[A^{*}\right]=\frac{\sqrt[x]{I_{A x B y}}}{\left[B^{\prime}\right]^{y}}=\frac{\sqrt[p]{J_{A p C^{\prime} z}}}{\left[C^{\prime}\right]^{2}}
$$

oder

$$
\left[\mathrm{B}^{\prime}\right]^{\mathrm{y} / \mathrm{x}}=\mathrm{K}\left[\mathrm{C}^{\prime}\right]^{\frac{z}{\mathrm{p}}}
$$

3) Tatsăchlich müssen wir hier $x$ an Stelle von $\frac{x}{m}$ schreiben, im $\$ 4$ ist aber auseinandergesetzt, dab wir hier auch $\frac{x}{m}$ setzen konnen.

$$
\begin{gathered}
{\left[\mathrm{B}^{\prime}\right]=\mathrm{K}^{\prime}\left[\mathrm{C}^{\prime}\right]^{\frac{\mathrm{z}}{\mathrm{p}}} \cdot \frac{\mathrm{x}}{\mathrm{y}}} \\
\frac{\mathrm{x}}{\mathrm{m}}=\alpha \mathrm{C}^{\frac{1}{\mathrm{n}}} .
\end{gathered}
$$

Die Größe des Koeffizienten $\alpha$ hängt also ab vom Vernaltnis der lonenprodukte der schwerlöslichen Stoffe; der Exponent 1/n ist abhängig von der Wertigkeit der reagierenden lonen. Für gleichwertige lonenhat $1 / n$ denselbe n Wert. Diese Schubfolgerung ist eine der wichtigsten aus der chemischen Erklärung der Elektroadsorption. Mit Hilfe der chemischen Auffassung muß man alle Erscheinungen der Elektroadsorption erklären können. Aus dem Obenstehenden geht $u$. a. deutlich hervor, das man die Elektroadsorption etwa mit der sog. Permutitwirkung vergleichen kann. Die Adsorption von Elektrolyten durch Kohle ist ein analoger Vorgang wie z. B. die Umwechselung von Basen durch Permutite.

DaB bei kleinen Endkonzentrationen relativ grote Mengen des adsorbierten Ions festgelegt werden, ist chemisch einfach abzuleiten. Daß nach Erreichung einer bestimmten Endkonzeritration die Menge des adsorbierten Stoffes nur wenig mehr zunehmen wird, ist zwar physikalisch nicht, wohl aber chemisch einfach abzuleiten. In Hinsicht auf die Permutitwirkung müssen wir auch chemisch ableiten können, dab das Festlegen von Wasserstoffionen durcí ein Aluminiumsilikat gemäB der Adsorptionsisotherme vor sich geht. Die stattfindende Reaktion können wir folgendermaBen zum Ausdruck bringen:

$\mathrm{Al}_{\mathrm{x}}(\text { Silikat })_{\mathrm{y}}+3 \mathrm{~b}[\mathrm{H} \cdot] \rightarrow \mathrm{xAl} \cdots+3 \mathrm{H}_{\mathrm{m}}(\text { Silikat })_{\mathrm{z}}$ $[\text { AI }]^{\mathbf{x}}\left[\right.$ Silikat $^{\mathrm{y}}=\mathrm{I}_{\mathrm{As}}$.

So ist auch

$$
\left[\mathrm{H}^{\cdot}\right]^{\mathrm{m}}[\text { Silikat }\}^{\mathrm{z}}=\mathrm{I} \mathrm{Bs} \text {. }
$$

Nun ist

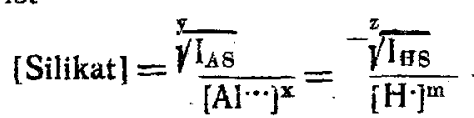

Hieraus können wir ableiten, dab

$$
\left[A I^{\cdots}\right]=\mathrm{K}\left[\mathrm{H} \cdot \frac{\mathrm{my}}{\mathrm{zy}}\right. \text {. }
$$

[H'] ist die Endkonzentration c. Da die Menge Wasserstoffionen, die fortgenommen werden, dreimai gröber ist als die Menge der gebildeten H-Ionen, so ist die Konzentration der letzteren 1/3 der Menge der fortgenommenen Wasserstoffionen, also

oder

$$
[\mathrm{A} \cdots]=1 / 3 \mathrm{x} / \mathrm{m} 1
$$

$$
\frac{\mathrm{x}}{\mathrm{m}}=3 \mathrm{Kc} \mathrm{c}^{-\frac{\mathrm{my}}{\mathrm{x} x}}
$$


oder

$$
\frac{\mathrm{x}}{\mathrm{m}}=\alpha \mathrm{c}^{1 / \mathrm{n}}
$$

Auch hier genügt die chemische Erklärung.

Eine andere Frage, auf die die chemische Erklärungsmethode eine Antwort zu geben vermag, ist die, warum Wasserstoffionen die Adsorption von sauren Farbstoffen im allgemeinen befördern, Hydroxylionen die von basischen.

Wenn wir von basischen Farbstoffen annehmen, dab der Farbstoff als Silikat festgelegt wird, dann wird dieser Lack durch Wasser stark hydrolytisch gespaiten werden, da die Kieselsäure $\mathrm{H}_{x} \mathrm{Si}_{y} \mathrm{O}_{z}$ sich wie eine sehr schwache Säure verhält, dit überdies schwerlöslich ist. Wenn man nun zu einer derartigen Lacksuspension Lauge hinzufingt, wird die Hydrolyse zurückgedrängt, so daßs mehr Silikat gebunden wird. $\mathrm{Ob}$ umgekehrt Wasserstoffionen die Aufnahme von Parbstoffionen merklich herabsetzen, hängt vom Ionenprodukt der Kieselsäure und des Farbstofflackes ab. Umgekehrt werden Wasserstoffionen die Fixierung von Farbstoffen durch positive Adsorbentien, wie Aluminiumoxydhydrat befördern und zwar aus denselben Gründen wie oben abgeleitet ist.

Die chemische Theorie ist somit imstande auf mehrere Fragen Antwort zu geben. Wir werden diese indessen nicht im einzelnen behandeln, sondern experimentell einen $\mathrm{Bew}$ e is für die chemische Auffassung zu liefern suchen. Bevor das jedoch geschieht, sei einmal in kurzen Zügen eine Uebersicht über die Auffassungen gegeben, die bislang über die chemische $\mathrm{Er}$ klärung der Adsorption im allgemeinen bekannt geworden sind.

3. Im Jahre 1908 veröffentlichte Brailsford Robertson ${ }^{4}$ ) unter dem Titel . Einige kritische Bemerkungen zur Theorie der Adsorption " eir e Arbeif, worin er alle Erscheinungen der Adsorption chemisch zw erklären suchte. Die Adsorption kann man nach diesem Verfasser ausdrücken durch die Gleichung:

$$
n_{1} A+n_{2} B \longleftarrow n_{3}(A B)
$$

worin $A$ das Adsorbens und $B$ den adsorbierten Stoff bedeutet. Da nach Ro berts on die Konzentration des Adsorbens lonstant ist, kann man mit Hilfe des Massenwirkungsgesetzes die Gleichung der Adsorptionsisothermen ableiten. Er vergleicht die Adsorption mit der Reaktion zwischen Essigsäure und Alkohol. Nach Robertson ist nie ein Beweis geliefert worden, die Adsorption durch Oberflächenverdichtung zu

4) T. B. Robertsor, Koll.-Zeitsehr. 3, 49 (1908). erklären, dahingegen ist nach ihm die Adsorption von Wasserstoff durch Palladium oder von Sauerstoff durch Platin sicher einem chemischen Vorgange zuzuschreiben. Nach Roberts on bedingt nun die eigenartige Struktur des Kohlenstoffatoms, daB der Kohlenstoff sich mit allerlei anderen Stoffen verbinden kann. Diese Auffassung wurde kürzlich erneut von $\mathrm{Mich}$ aelis und Ron a ${ }^{5}$ ) verkündet, deren Anschauungen ich hier unbesprochen sein lassen will, weil ich mich mit vielen derselben nicht einverstanden erklären kann.

Ferner bespricht $\mathrm{R}$ obe $\mathrm{r} t \mathrm{~s}$ o $\mathrm{n}$ die Bedeutung seiner Anschauung für die Immunchemie und Biochemie. Seine Ansichten in dieser Hinsïcht werden jedoch von $L$ andste in e $r^{6}$ ) und Bayli s s ${ }^{7}$ ) bestritten:

Die Behauptungen von Robertson hat am eingehendsten $\mathrm{Freund}\left(\mathrm{Ch}^{8}\right.$ ) kritisiert. In erster Linie stellte Roberts on die Kriterien der Adsorption unrichtip dar, und ferner ist die Ableitung seiner Gleichung falsch. Es scheint mir außerdem sehr gewagt, anzunehmen, dab die sehr indifferente Kohle sich mit den verschiedensten Stoffen "verbinden " kann. Nach Freundlich ist es im allgemeinen unmöglich, das Gleichgewicht der Adsorption mit Hilfe des Massenwirkungsgesetzes abzuleiten. Wie wir gesehen haben, ist das nicht richtig. Auch die Behauptung von Freundlich, dab eine typische Eigenschaft der Adsorption darin bestehen soll, dafi gerade bei kleinen Enăkonzentrationen relativ sehr viel adsorbiert wird, ist rom chemischen Standpunkte aus ebenfalls einfach zu erklären, wie aus den angegebenen obigen Betrachtungen abgeleitet werden kann

Ein anderer Versuch, die Adsorption im allgemeinen rein chemisch $z u$ erklären, wurde von Reychler ${ }^{9}$ ) gemacht. Biltz ${ }^{10}$ ) hatte nämlich abgeleitet, daß bei der Adsorption von Arsentrioxyd durch Ferrioxydhydrat zwischen der adsorbierten Menge $\mathrm{As}_{2} \mathrm{O}_{3} \mathrm{y}$ und der in Lösung gebliebenen Menge $\mathrm{x}$ das folgende Verhältnis besteht:

$$
\frac{y^{5}}{x}=0,63
$$

5) L. Michaelis u. P. Rona, Biochem. Zeitschr. 97, 57 (1919).

6) K. Land ste iner, Koll.-Zeitschr. 3, 221 (1908).

7) W. B ay lis s, Koll.-Zeitschr. 3, 224 (1908).

8) H. Freund $1 \mathrm{ich}$, Koll.-Zeitschr. 3, 212 (1908). (1909.)

9) A. Reychler, Joum. d. chim. Phys. 7, 362 (1904).

io) W. B11tz, Ber. d. Deutsch. chem. Ges. 37, 3138 
Reychler nimmt nun an, daß die Verbindung Ferriarsenit gebildet wird, die zum groben Teile hydrolysiert ist. Er leitet alsdann $a b$, dab man hier die Beziehung findet

$$
\frac{y^{m}}{x}=K \text {. }
$$

B i $1 \mathrm{tz}^{11}$ ). bestreitet die Auffassung von Reychler. Nach ihm darf man das Massenwirkungsgesetz auf ein derart inhomogenes System nicht anwenden. Ferner verhält arsenige Såure sich nicht als dreiwertige Säure. Diese beiden Bemerkungen von Biltz sind m. E. einfach zu widerlegen, von gröberer Bedeutung ist indessen seine Bemerkung, daß man mit Hilfe der Hydrolyse die Erscheinungen nicht erklären kann. In diesem Falle soll die Adsorption sich nämlich stark mit der Temperatur ändern, was nicht so ist. Aber nun braucht man auch nicht anzunehmen, daß das chemische Gleichgewicht durch die Hydrolyse beherrscht wird. Das Eisenoxydhydrat in einem Eisenoxydsol enthält auch stets Chlorid, das wahrscheinlich komplex an Eisen gebunden ist: Wir können also auch annehmen, dab Ferrioxychlorid sich mit der arsenigen Säure verbindet. Aber auch, wenn wir das nicht annehmen, und voraussetzen, daB das Ferriarsenit direkt aus Eisenoxydhydrat und Arsentrioxyd entsteht, ist es nicht notwendig, daB das Gleichgewicht bei hoher Temperatur sich stark ändert. Zwar nimmt die Hydrolyse stark $z u$, doch ist es auch unbestimmt, auf welche Weise die Stabilität der Verbindung sich ändert. In einer weiteren Mitteilung leitet Reychler ${ }^{12}$ ) ab, daB man die Adsorption von arseniger Säure durch Kohle ebenfalls rein chemisch erklären kann. Mit Hilfe der von $\mathrm{Fr}$ u $\mathrm{ndlich}$ bestimmten Zahlen leitet er für jede Säure eine Konstante $a b$. So wichtig im allgemeinen seine Ansichten auch sein mögen, so ist es doch bedauerlich, daß in seiner Ableitung ein Fehler steckt. Freundlich ${ }^{13}$ ) kann sich dann auch nicht mit der chemischen Auffassung einve rstanden erklären.

Wir sehen also, dab es noch nicht gelungen ist, die ganze Adsorption nur vom chemischen Standpunkte aus zu erklären. Dies scheint mir aber auch nicht nötig und selbst nicht möglich. DaB kapillaraktive Stoffe wie Alkohole, Phenole usw. durch Oberflächenverdichtung adsorbiert werden, scheint mir seìr verständlich. Es ist

1i) W. Biltz, Journ. d. chim. Phys. 7, 570 (1909). (1909)

A. Reychler, Journ. d. chim. Phys. 7, 497

13) H. F r e u nd li c h, Zeitschr. f. physik. Chem. 73, 385 (1910.) darum aber nicht unmöglich, dab dabei auch ein chemischer Vorgang eine Rolle spielt, aber diese Frage wird vom stöchiochemischen Standpunkte aus sehr schwierig. Wir kennen dann nämlich nicht mehr die Konzentration des reagierenden Stoffes in der Nähe der Kohleoberfläche und die Art der Verbindung, die sich bildet.

Nach der anderen Seite hin sind die Erscheinungen der Elektroadsorption derartig, da wohl niemiand mehr versuchen wird, sie physikalisch durch Oberflächenverdichtung zu erklären. Hier verdient die rein chemische $\mathrm{Er}$ klärungsmethode als die richtigste angenommen zu werden, um so mehr als sie, wie wir sehen werden, uns ein Arbeitsgebiet öffnet, auf dem wertvolle Ergebnisse erhalten werden können.

4. Eine grobe Schwierigkeit, die gegen die chemische Auffassung besteht, haben wir noch nicht besprochen. Bei der chemischen $A b$ leitung der Gleichung der Adsorptionsisotherme haben wir angenommen, dab die Menge Ionen, die bei der Adsorption verdrängt wird, nämlich $x$ (siehe unter 2), in gewohnter Weise auf die Menge, welche für $1 \mathrm{~g}$ Adsorbens verdrängt wird $\left(\frac{x}{m}\right)$, umgerechnet werden kann. Gegen das letztere besteht nun, wie es scheint, eine grobe Schwierigkeit. Wenn wir nämlich wieder den einfachen Fall nehmen, daß wir Silberbromid mit Rhodanid schütteln, dann ist es gleichgültig, wieviel Silberrhodanid anwesend ist. Die Menge Bodenkörper spielt bei der Einstellung des Gleichgewichtes keine Rolle, während bei einer Adsorption desto mehr fort genommen wird, je mehr Adsorbens vorhanden ist. Hier müssen also die Gleichgewichtskonzentrationen von der Menge des Adsorbem (Bodenkörper) abhängig sein, was in erster ln. stanz mit dem Massenwirkungsgesetz in Wider spruch zu stehen scheint.

Dieser Widerspruch ist indessen nur scheinbar. Zwar wird durch die Rolle, welche die Menge Oberfläche (Menge Adsorbens) spieit die Sache komplizierter, aber die obigen Ausführungen werden nicht wertlos dadurch. Im Gegenteil, wir können die Erklärungen im Anschlub an die Ableitungen nach dem Massen. wirkungsgesetz geben. Wie aus den Untersuchungen von Perrin u. a. hervorgeht, sind verschiedene Stoffe wie Chromoxyd, Karborundum, Kohle, Quarz, Glas u. dgl. in Wasser elektrisch geladen. Ein derartiges Teilchen verhält sich als elektrischer Doppelkondensator. Die Ladung des Teilchens wird beherrscht von 
den lonen, die an der Oberfläche ihren Sitz haben. Meiner Ansicht nach ist es dabei erforderlich, da eine bestimmte (chemische) Affinität besteht $z$ wischen dem festen Teilchen und dem lon, das direkt an seiner Oberflache festgelegt wird. Es soll gewöhnlich ein lon sein; das das Teilchen selbst liefern kannodermit dem es einen Komplex bildet. Nur hierdurch kan man alleineinsehen, warum ein Teilchen gerade eine Vorliebe für das lon zeigt, das an seiner Oberfläche sitzk, das alsodieLadung bestimmt. Wenn wir z. B. ein Kieselsäureteilchen betrachten, dann hat dieses an seiner Oberfläche Silikationen adsorbiert, die dem Teilchen seine negative Ladung geben. Darum herum sitzen in der doppelten Schicht die indifferenten lonen, in diesem Falle Wasserstoffionen oder wahrscheinlicher Kalium- oder Natriumionen, herrührend von Verunreinigungen. Dadurch, daB das Teilchen also elektrisch geladen ist, befindet sich an seiner Oberfläche eine grobe Konzentration von Silikationen, welche bei der Adsorption gerade die aktive Rolle spielen. Was von der Kieselsăure gesagt ist, gilt eben so sehr von anderen Adsorbentien und auch von Suspensionskolloiden. So nimmt Zsigmondy an, daß das Zinnoxydsol durch Stannationen stabilisiert wird; ferner scheint mir wahrscheinlich, daß ein Arsentrisulfidsol durch Bildung komplexer Sulfarsensäureionen stabilisiert wird, was ich weiter unten noch näher erläutern will.

Wenn wir uns nun die "Adsorption" von Elektrolyten, z. B. von Kieselsäure, näher anseheñ, dann können wir einfach ableiten, daß die Menge Kieselsäure ( "Adsorbens"), d. h. auch die Totalmenge oder GröBe der Oberfläche eine Rolle spiclt. Kieselsăure ist in Wasser praktisch völlig unlöslich. Eine wässerige Lðsung von Kieselsäure reagiert dann auch nicht mit basischen Farbstoff- oder anderen Kationen, weil die Konzentration der Silikationen vernachlässigbar klein ist. Anders verhält es sich an der Oberfläche des Kieselsäureteilchens. Wie bereits gesagt ist, wird die Ladung des Teilchens durch die Silikationen beherrscht, die an der Oberfläche sitzen. Diese sind hier also in großer Konzentration anwesend (alle Teilchen sind bestrebt, sich in Wasser zu einem Potential von $70 \mathrm{~m}$-Volt aufzuladen). Wenn ${ }^{*}$ wir nun die Kieselsäure mit einer Lösung einer Parbstoffbase in Berührung bringen, dann diffundiert letztere an die Oberfläche des Teilchens. Hier trifft sie eine große Konzentration von Silikationen an, mit denen sie chemisch gemäß dem Massenwirkungsgeset $z$ unter Bildung des Farbstofflackes in Reaktion tritt. Gleichzeitig hiermit nimmt die Ladung des Teilchens ab oder, wie wir sagen können, findet die Adsorption statt. Der Farbstofflack wird an der Oberfläche des Teilchens niedergeschlagen und macht hierdurch die Oberfläche weiterhin inaktiv. Wenn wir nun mit Farbstoff im Ueberschub arbeiten, dann stellt sich schlieblich an der Oberfläche ein Gleichgewicht ein, wobei der FarbstoffionenüberschuB sich mit einer sehr geringen Konzentration von Silikationen im Gleichgewicht befindet (die Konzentration der letzteren hängt natürlich vom lonenprodukt der genannten Verbindung $a b)$. Wenn wir von neuem Kieselsäure zufügen, dann kommt der gelöste Farbstoff wieder in Berührung mit der Grenzschicht, wo sich eine viel größere Konzentration von Silikationen befindet, als sich mit der vorhandenen Menge Farbstoffionen im Gleichgewicht befinden kann. Die beiden lonen werden also von neuem miteinander reagieren, bis sich das Gleichgewicht wieder eingestellt hat. Hierdurch wird also die Rolle der Menge des Adsorbens aufgeklärt.

Zugleich mit der chemischen Reaktion findet also auch die Entladung der Wand statt. Beide Erscheinungen laufen parallel. Da die Aenderung in der Menge des adsorbierten lons mit der Konzentration durch die Gleichung der Adsorptionsisothermen beherrscht wird, ist auch zu erwarten, daf die Verminderung der Ladung im Hinblick auf die Konzentration des adsor bierten" Stoffes durch eine analoge Gleichung ausgedrückt wird. In der Tat geht aus Versuchen von $\mathrm{Kruyt^{14 }}$ ) u. a. hervor, daB das der Fall ist.

Die Rolle der Oberfläche wird hier also durch die eigenartige Beschaffenheit der Grenzschicht erklärt. Jedes Teilchen des Adsorbens ist durch seine elektrische Ladung gegenüber der Flüssigkeit in einen besonderen Zustand gebracht. Das aktive lon, das bei der Adsorption die Rolle spielt und die Ladung des Teilchens beherrscht, ist in der Grenzschicht in viel grögerer Konzentration anwesend als in der Flüssigkeit. Wenn die Reaktion (Adsorption) stattfindet, wird die Oberfläche inaktiviert und die Konzentration der aktiven lonen wird sehr klein. Wenn die reagierenden (adsorbierten) lonen nun wiedar mit einer frischen Oberfläche

14) H. R. Kr uyt, Koll.-Zeitschr. 22, 81 (1918). 
in Berübrung kommen, treffen sie hier eine viel größere Konzentration an aktiven lonen an, als sich mit ihnen im Gleichgewicht befinden. Es wird von neuem eine Reaktion stattfinden, und das wird sich so oft wiederholen, als wir eine aktive Oberfläche mit der Flüssigkeit zusammenbringen.

5. Experimenteller Teil. Wie wir oben abgeleitet haben, ist der Wert von $1 / n$ in der Gleichung $\frac{x}{m}=\alpha \frac{l}{c^{n}}$ van grober Bedeutung, da er von der Wertigkeit der reagierenden Stoffe beherrscht wird. Wie wir wus den obigen Betrachtungen direkt ableiten konnten, mub eine einfache Beziehung bestehen zwischen der Wertigkeit des adsorbierten lons und $1 / n$. Wenn $z$. B. $1 / \mathbf{n}$ für ein einwertiges Ion gleich $a$ ist, dann mub es für ein zweiwertiges Ion $1 / 2$ a, für ein dreiwertiges $1 / 3$ a sein.

Es sind nun in der Literatur sehr viel Adsorptionsversuche beschrieben; doch liefern dieselben nicht hinreichendes Material, um das Obenstehende zu prüfen. Stets mut man auch die Versuche miteinander vergleichen, die mit derselben Kohlesorte angestellt sind. Zudem sind die Ergebnisse der Versuche, die mit sauren Metallsalzen angestellt wurden, wegen der groBen Analysenfehler unbrauchbar.

Wohl folgt aus verschiedenen Versuchen in der Literatur, das $1 / \mathrm{n}$ für gleichwertige Stoffe wenig veränderlich ist ${ }^{15}$ ). Nur eine Veröffentlichung gibt eine schöne Bestätigung des von mir vertretenen Standpunktes, nämlich eine $A b$ handlung von Freundlich ${ }^{16}$ ) im Jahre 1910, worin er sich gerade noch gegen die chemische Auffassung der Adsorption ausspricht.

Seine Zahlen von $1 / n$ stimmen schön mit der oben genanuten Auffassung überein.

Freundlich gebrauchte aus besonderen Gründen Arsentrisulfid als Adsorbens. In der folgenden Tabelle gebe ich die Werte wieder, die er von verschiedenen Elektrolyten fand:

\begin{tabular}{c|l|l}
\hline $\begin{array}{c}\text { Wertigkeit } \\
\text { des Kations }\end{array}$ & Adsorbierter Stoff & $1 / n$ \\
\hline ein & Anilin(chlorid) & 0,208 \\
ein & p-Chloranllin & 0,194 \\
ein & n-Fuchsin & 0,194 \\
ein & Ammoniumchlorid & 0,2034 \\
ein & Ammoniumsulfat & 0,1831 \\
\hline zwei & Uranyl & 0,0954 \\
drei & Cero & 0,077 \\
\hline
\end{tabular}

15) Siehe H. Freundlich, Kapillarchemie 149, auch Tabelle S. 150, 151.
Die Zahlen von Freundlich bedürfen keiner weiteren Erläuterung. Es kann nicht Zufälligkeit sein, dab der Wert vọn $1 / \mathrm{n}$ von so verschiedenen einwertigen Ionen, wie sie Freundlich anwendet, dieselbe ist. AuBerdem ist $1 / \mathrm{n}$ vom $z$ weiwertigen Uranyl die Hälfte des Wertes von einwertigen lonen und von Cero ein Drittel.

Kürzlich ist der Anfang einer Serie von Untersuchungen erschienen, die Sven $\mathrm{Odé}{ }^{17}$ ) in Gemeinschaft mit Hugo Andersson über. den Einflub der Stōchiometrie bei der Adsorption angestellt hat. Dieselben nahmen die Versuche mit besonders reinen Kohlesorten vor und bestimmten die Adsorptionsisotherme von verschiedenen Salzen. Die Analyse führten sie mittelst des Interferometers aus. Wenn die Elektroadsorption wirklich auf einer Verdrängung eines lons an der Oberfläche eines Adsorbens durch ein anderes beruht, ist die An= wendung dieser Methode beim Arbeiten mit Ionen mit kleiner spezifischer Refraktion nicht ohne Bedenken. Dennoch gebe ich in der fol. genden Tabelle die Werte von $1 / n$, die die Verfasser bei verschiedenen Salzen fanden, wieder:

\begin{tabular}{|c|c|c|c|}
\hline Salz & Kohle I & Salz & Kohle II \\
\hline \multirow{2}{*}{$\begin{array}{l}\mathrm{CsNO} \\
\mathrm{RbNO}_{3} \\
\mathrm{NH}_{4} \mathrm{NO}_{8} \\
\mathrm{KNO}_{3} \\
\mathrm{NaNO}_{3}\end{array}$} & \multirow{2}{*}{$\begin{array}{l}0,81 \\
0,81 \\
0,86 \\
0,90 \\
0,95\end{array}$} & $\begin{array}{l}\mathrm{KNO}_{3} \\
\mathrm{NaNO}_{8} \\
\mathrm{LNO} \mathrm{O}_{8}\end{array}$ & $\begin{array}{l}0,78 \\
0,86 \\
0,87\end{array}$ \\
\hline & & $\begin{array}{l}\mathrm{Ba}\left(\mathrm{NO}_{3}\right)_{2} \\
\mathrm{Sr}\left(\mathrm{NO}_{8}\right)_{2} \\
\mathrm{Ca}\left(\mathrm{NO}_{3}\right)_{2} \\
\mathrm{Mg}\left(\mathrm{NO}_{3}\right)_{2}\end{array}$ & $\begin{array}{l}0,45 \\
0,50 \\
0,63 \\
0,58\end{array}$ \\
\hline
\end{tabular}

Obwohl hier also der Wert von $1 / \mathrm{n}$ bei der Adsorption von den zweiwertigen Kationen nicht genau die Halfte des bei einwertigen lonen gefundenen betragt, ist der Unterschied nicht so groB, das man das Ergebnis als mit der chemischen Auffassung der Elektroadsorption in Widerspruch stehend ansehen kann.

Um mehr Angaben zu besitzen, habe ich eine Serle Prüfungen mit Noritkohle vorgenommen, die zuvor zwecks Reinigung mit Salzsäure ausgewaschen war. Die Analysenmethoden und Analysenergebnisse, die sehr zuverlässig sind, will ich hier der Kürze wegen nịcht

16) H. Fre und lich, Zeitschr. f. physik. Chem. 73. 385 (1910).

17). Sven Odén u. Hugo Andersson, Journ. Physic. Chem. 25, 311 (1921). 
beschreiben, sondern nur die erhaltenen Werte von $\frac{1}{n}$ mitteilen ${ }^{18}$ ).

Als zweiwertige lonen wurden Kupfer und Blei genommen. Als einwertige lonen wurden verschiedene Alkaloidsalze untersucht. Auch wurden einzelne zweiwertige Alkaloidsalze untersucht, nämlich Chiriinchlorid und Strychninnitrat. Bei den letzteren ergaben sich UnregelmäBigkeiten, da bei einer bestimmten Endkonzentration ein Maximum in der Menge des adsorbierten Stoffes auftritt. Dies ist keine $\mathrm{Zu}$ fälligkeit, da F r e u nd l i c h ${ }^{19}$ ) von S try ch n innitrat dasselbe Verhalten festgestellt hat. Wahrscheinlich wird die Störung dadurch verursacht, dab sowohl ein- als zweiwertige lonen adsorbiert werden. Aber dadurch wird das hydrolytische Gleichgewicht in der Flüssigkeit verschoben, so dab auBerdem die Möglichkeit besteht, dab Hydroxylionen frei kommen und adsorbiert werden. Eine ausführlichere Untersuchung soll aber dazu dienen, herauszubringen, ob die Sache nicht von tieferer Bedeutung ist.

In der folgenden Tabelle gebe ich nur die Ergebnisse wieder, die ich für $\frac{1}{n}$ fand.

\begin{tabular}{l|l|l}
\hline Wertigkeit & Adsorbiertes Ion & $1 / \mathbf{n}$ \\
\hline zwei & Kupfer (Sulfat) & 0,167 \\
zwei & Blei (Nitrat) & 0,16 \\
ein & Morphin (Chlorid) & 0,303 \\
ein & Codein (Chlorid) & 0,308 \\
ein & Papaverin (Chlorid) & 0,302 \\
ein & Atropin (Sulfat) & 0,28
\end{tabular}

Die Uebereinstimmung mit der Theorie ist also vorzüglich.

Auch habe ith noch einige Adsorptionsversuche mit Anionen ausgeführt. Wegen der Genauigkeit, mit der diese Ionen bestimmt werden können, habe ich die folgende gewählt:

\begin{tabular}{l|l|l}
\hline Wertigkeit & Adsorbiertes Ion & $1 / \mathbf{n}$ \\
\hline \hline drei & Ferrizyanid & 0,04 \\
zwei & Oxalat (Kalium) & 0,08 \\
zwei & Chromat (Kalium) & 0,06 \\
ein & Jodat (Kalium) & 0,16
\end{tabular}

6. Wir haben bis jetzt gesehen, dab die chemische Theorie der Elektroadsorption nicht allein

18) A. a. O. werde ich die Ergebnisse ausführlich mitteilen.

19) H. Freund l i ch, Zeitschr.f. physik. Chem. 73, 385 (1910). imstande ist, die Erscheinungen, die sich bei der Adsorption zeigen, zu erklären, sondern daß sie auch auberdem noch eine RegelmäBigkeit in den Beziehungen zwischen $\frac{1}{n}$ und der Wertigkeit des fixierten Ions voraussagen lieb, die durch die Proben bestätigt wurde. Eine weitere Frage ist nun: „Welche Aussichten eröffnet uns nun die chemische Theorie." Ich will nun von einzelnen Fällen deren Bedeutung näher ausführen.

a) Die Elektroadsorption von $z$ wei lone n nebeneinander: Bei gleichzeitiger Adsorption von zwei fonen kann man natürlich empirisch bestimmen, welche Beziehung besteht zwischen den Mengen, die fixiert werden und ihren zugehörigen Endkonzentrationen in der Flüssigkeit. Wenn man aber die verschiedenen Konstanten der gebildeten Verbindungen kennt, karn man auf rein chemischem Wege auch berechnen, wie die Verteilung der fixierten Ionen an der adsorbierenden Oberfläche stattfindet.

b) Adsorptionsverbindungen. Es ist sowohl von praktischem als auch theoretischem Werte, die ,Adsorptionsverbindungen ${ }^{*} z \mathfrak{u}$ untersuchen. Es wird sich m. E. zeigen, dab diese Adsorptionsverbindungen rein chemische Verbindungen sind, obwohl ihre Zusammensetzung stark wechseln kann. Auch bei Vorgängen, die rein chemisch verlaufen, erhält man häufig Reaktionsprodukte von wechselnder Zusammensetzung, die Gemenge voin Stoffen bestimmter stöchiometrischer Zusammensetzung sind. So wird wohl niemand die Verbindungen, die sich niederschlagen, wenn man Sublimat mit Ammoniak und Ammoniumchlorid behandelt, Adsorptionsiverbindungen nennen wollen. Ebensowenig wird man Gemenge von neutralen und basischen Salzen (verschiedene Magnesiumkarbonate) als Adsorptionsverbindungen ansehen. Wenn man die Eigenschaften der Bestandteile, die einen derart verwickelten Komplex bilden, kennt, kann man von vornherein die Zusammensetzung des Reaktionsproduktes prophezeien. Wenn man nun z. B. ein Aluminiumsalz mit einem sauren Farbstoff behandelt, ist es nicht nötig, daß nur eine Verbindung vom Typus $\mathrm{AlZ}_{3}$ ausfällt; gleichzeitig wird auch z. B. AlOHZ, und dgl. entstehen können. Dies hängt ganz ab von den Eigenschaften, Ionenprodukt und Hydrolysengrad der verschiedenen Verbindungen. Es ist also nicht erlaubt zu sagen, dab die stöchiometrischen Gesetze nicht mehr anzuwenden. seien, wenn die Zusammensetzung der genannten Verbindungen wechselnd $z u$ sein scheint. Es 
ist nötig, die zugehörigen Verbindungen von bestimmter stöchiometrischer Zusammensetzung abzuscheiden und ihre Eigenschaften im Verein mit denen der Komponenten zu studieren. Dann kann man von vornherein sagen, welche Verbindungen, unter bestimmten Umständen entstehen werden.

c) Dèr Färbungsvorgang. Ich betrachte die Fixierung eines Farbstoffes auf der Faser ebenfalls als rein chemischen Vorgang. Nicht die reine pflanzliche oder tierische Faser legt den Farbstoff fest, sondern die Verunreinigungen. Ich will hier nicht die Theorie des Färbungsvorganges ausfiuhrlich besprechen. Untersuchungen, die ich mit Zellulose (Filtrierpapier und Watte) mit den verschiedensten Elektrolyten anstellte, führten zu dem Ergebnis, dab alle Kationen in äquivalenten Mengen festgelegt werden, und zwar war diese Menge gleich der Alkalität der Papierasche. Wurde das Papier gereinigt, so daß sich die Alkalität verminderte, so nahm die Fähigkeit, Kationen zu fixieren, in gleichem MaBe ab.

Es scheint mir nun für die praktische Entwicklung des Färbungsvorganges von großer Bedeutung, då man die Verbindungen, die sich auf der Faser bilden, zu isolieren and ihre Eigenschaften festzustellen sucht. Auch die Verbindungen, die die Farbstoffe mit verschiedenen $B$ eizen bilden, muB man isolieren und ihre Eigenschaften vom physikalisch - chemischen Standpunkt aus studieren. Kennt man diese, dann kann man die Umstände ableiten, bei denen man unter den sparsamsten und zweckmäBigsten Bedingungen das Verfahren des Färbens ausführen kann.

d) Suspensionskolloide. Obwohl man weiB, dab die Suspensionskolloide ihre Stabilităt kleinen Mengen von adsorbierten Elektrolyten verdanken, weib man nicht, auf welche Weise diese festgelegt sind. Zwar nimmt man z. B. im Falle von Arsentrisulfidsol an, daB dieses seine Stabilität adsorbiertem $\mathrm{H}_{2} \mathrm{~S} z u$ danken hat, daß die Schwefelionen an der Oberfläche der $\mathrm{As}_{2} \mathrm{~S}_{3}$-Teilchen sitzen und die Wasserstoffionen sich in einer elektrolytischen Doppellage darum herum befinden, doch befriedigend ist diese Erklärung keineswegs; diese Vorstellung ist sogar sehr unwahrscheinlich:

Vernünftiger erscheint es, anzunehmen, $\mathrm{da}$ die unlöslichen Teilchen, im vorliegenden Falle die $\mathrm{As}_{2} \mathrm{~S}_{3}$-Teilchen, sich mit dem UeberschuB des zugefügten Elektrolyten verbinden, bei Arsentrisulfid mit $\mathrm{H}_{2} \mathrm{~S}$. Die $\mathrm{As}_{2} \mathrm{~S}_{3}$-Teilchen bilden dann mit den $\mathrm{S}$ :lonen eine komplexe
Arsenschwefelverbindung. Auch Reychler ${ }^{20}$ ) nimmt dies letztere an. $\mathrm{Er}$ gibt an, daB Verbindungen des Typus $\mathrm{OH}-\mathrm{As}=\mathrm{S}_{2}=\mathrm{As}-\mathrm{OH}$ oder $\mathrm{HS} \quad \mathrm{As}=\mathrm{S}_{2}-\mathrm{As}$ - HS oder $(\mathrm{OH})_{2}$ $=A s-S-A s-(S H)_{2}$ usw. gebildet werden, die selbst wenig löslich sind. Es ist eine lange bekannte Tatsache, daB $\mathrm{As}_{2} \mathrm{~S}_{3}$ Neigung besitzt, mit Schwefel verschiedene Komplexe zu bilden, wofür das Bestehen verschiedener Schwefelarsen(Sauerstoff-)Verbindungen spricht. Man kann sich also vorstellen, dab die Reaktion wie folgi verläuft:

$$
\mathrm{xAs}_{2} \mathrm{~S}_{3}+\mathrm{yH} \mathrm{H}_{2} \mathrm{~S} \longleftrightarrow \mathrm{H}_{2 \mathrm{y}} A \mathrm{~s}_{\mathrm{x}} \mathrm{S}_{3 \mathrm{x}}+\mathrm{y} .
$$

In alkalischer Lösung sind diese komplexen Verbindungen beständig, in saurer Lösung wird das Gleichgewicht praktisch völlig nach links verschoben; aber es bleibt natürlich stets eine sehr kleine Menge des komplexen lons, das an der Oberfläche von $\mathrm{As}_{2} \mathrm{~S}_{3}$-Teilchen sitzt. Wenn man sich vorstellt, dab die komplexen Ionen an der Oberfläche der $A s_{2} S_{3}$-Teilchen sitzen, dann können die Wasserstoffionen in gewöhnlicher Weise in der Flüssigkeit verteilt sein. Diese Auffassung stimmt auch mit dem elektrischen Verhalten eines Arsentrisulfidsols überein und schließt sich den Auffassungen von Duclaux ${ }^{21}$ ) an. Das Ausflocken von Arsentrisulfidsol durch Elektrolyte mub man mun durch die Annahme erklären, dab die stabilisierenden, komplexen Sulfarsenigsäureionen von den ausflockenden lonen fortgenommen werden. Durch Wasserstoffionen wird die Spaltung der komplexen Verbindung in $\mathrm{As}_{2} \mathrm{~S}_{3}$ sehr stark befördert, weshalb Wasserstoffionen ausflockend wirken. Von den anderen stark ausflockenden einwertigen lonen wie Silber, Anilin, Alkaloiden, muß man annehmen, daB sie schwer lösliche Verbindungen mit dem Sulfarsenigsäureion bilden. Von den zwei- und dreiwertigen Ionen gilt dasselbe. Von den Alkalimetallen ist der Ausflockungswert viel größer, weil sich ithre Verbindungen besser lösen.

Die gegebene Erklärung für das Ausflocken ist verständlicher als die bislang angenommene. Es ist nicht einzusehen, warum z. B. Bariumionen auf ein Arsentrisulfidsol stark ausflockend wirken. Auch wenn Bariumionen stark adsorbiert werden, ist noch nicht einzusehen, daB sie Sulfidionen fortnehmen sollen, da Bariumsulfid gut dissoziiert ist. Dasselbe gilt von verschiedenen anderen lonen. Ferner ist nicht

209 A. Reychler, Journ. d. chim. Phys. 7, 497

21) J. D u cl a ux, Journ. d. chim. Phys. 7, 405 (1909). 
einzusehen, daß Elektrolyte physikalisch adsorbiert werden, weil sie auf die Oberflichenspannung keine Wirkung ausüben.

Der Plockungswert eines lons hat theoretisch nun auch sehr wenig Bedeutung. Viel notwendiger ist es, für jedes ausflockende lon die Werte des Koeffi$z$ ienten der Adsorptionsisothermen $z u$ bestimmen. Hierbei wird man für gleichwertige lonen dasselbe $1 / n$ finden müssen.

Zwei Versuche wurden von Freundlich (1910) nach dieser Richtung hin mit $A s_{i} S_{y}$-Sol unternommen. Er fand tatsächlich für Morphin und Neufuchsin ungefähr dasselbe $1 / \mathfrak{n}$.

$\mathrm{Da}$ man es bei Solen wie von $\mathrm{As}_{2} \mathrm{~S}_{3}$ und dgl., mit einfacheren Körpern zu tun hat als mit Adsorbentien wie Kohle und dgl;, so ist hier gerade von grober Bedeutung, die oben genannten Versuche über die Adsorption zu nehmen. Von den meisten Solen weib man, welche Elektrolyten anwesend sind. Kennt man nun $1 / n$ von ver. schiedenen lonen, dann kannmanmit Wahrscheinlichkeit herausbringea; in welcher Form das stabilisierende lon adsorbiert" ist, oderwiedie Zusammensetzung des komplexen stabilisierenden lons ist.

Wenn man seine Untersuchungen a uf diese Weise anstellt, ist dia Hoffnung begründet, das nan die Eigen. schaften der Suspensionskolloide wieder mit Hilfe der Stöchiometrie erklären und ableiten kann.

Im vorstehenden habe ich versucht, so knapp wie möglich die praktische und theoretische Bedeutung der chemischen Theorie wiederzugeben. Da wir augenblicklich noch nicht über genügende Versuchsergebnisse verfügen, könnte eine ausführlichere Besprechung der Wichtigkeit der Theorie gefährlich sein, da man dann leicht unter der Einflub von phantastischen, noch nicht kontrollierbaren Ideen geraten könnte.

\section{Zusammenfassung.}

a) Die Elektroadsorption ist eine rein chemische Erscheinung. b) Die Adsorptionsisotherme kann einfach mit Hilfe der Stöchiomerrie abgeleitet werden, wenn man annimmt, dab die Elektroadsorption eine Erscheinung ist, bei der ein sthwerlöslicher Sioff, der sich im Adsorbens befindet, sich mit dem fixierten lon zu einem anderen schweriöslichen Stoffe ansetzen kann.

$$
\text { c) Der Exponent } \frac{1}{n} \text { der Adsorptions- }
$$

isothermesteht in direkter Beziehung $z$ u der Wertigkeit der reagierenden stoffe. Wenn $\frac{1}{n}$ eines einwertigen lons gleich a ist, dann ist es fur ein zweinertiges lon $\frac{a}{2}$ für ein dreiwertiges $\frac{8}{3}$. Sowohl aus Versuchen von Preundlich und von $S$ ven $O$ dén wie aus eigenen Versuchsergebnissen geht die Richtigkeit dieser Behauptung hervor.

4) Die Bedeutung der Menge des Adsorbens wird durch die eigenartige Grenzschichtbeschaffenheil von jedem Teilchen erklärt. Das aktive Ion, das in der Grenzschicht die Ladung bestimmt und bei der "Adsorption" die unlösliche Verbindung bildet, ist hier in viel größerer Konzentration vorhanden, als in der Flüssigkeit. Wenn sich das Gleichgewicht an der Oberflache eines Teilchens eingestellt hat, dann ist die Konzentration des aktiven Ions, das in Berührung mit dem Ueberschuß lons steht, klein. Bringt man nun wieder frisches Adsorbens zur Flüssigkeit, dann gelangen die reagierenden Ionen an der Oberfläche jedes Teilchens wieder in Berührung mit einer groben Konzentration von aktiven lonen. Die Reaktion wird also von neuem statfinden.

e) Die Bedeutung der chemischen Theorie für die Kolloidchemie im allgemeinen wird kurz besprochen.

Zum Schlufi ist es tuir ein Vergnügen, Herrn Prof. Dr. Kru y t meinen aufrichtigen Dank auszusprechen für das Interesse und die Anregung, die er mir bei der Bearbeitung dieser Mitteilung hat zuteil werden lassen.

Litrecht, September 1921.

Pharmazeutlsches Laboratorium der Universitat, 